Mehr Zirrhosen, Kolostomien \& Co.

\title{
Nach Krebs im Kindesalter spielt Magen-Darm-Trakt verrückt
}

> Erwachsene, die als Kind an Krebs litten und eine dementsprechende Behandlung, wie Chemotherapie, Radiotherapie oder chirurgische Interventionen erhielten, haben später ein erhöhtes Risiko für gastrointestinale Komplikationen. Dies ging aus einer Studie mit 14.358 Krebspatienten verschiedener Arten - z.B. Leukämie, Lymphomen oder Neuroblastomen - und deren Geschwistern hervor. Das mediane Erkrankungsalter der Betroffe-

\section{Bei Parkinson auf Melanome achten!}

> Nach Diagnose einer Parkinsonerkrankung laufen Betroffene Gefahr, zusätzlich ein malignes Melanom zu bekommen. Die Resultate einer Metaanalyse zeigten, dass das Risiko für ein Melanom nach Manifestation der degenerativen Hirnerkrankung um den Faktor 3,6 ansteigt. Als Ursache wird ein Zusammenhang mit der Synthese des Farbstoffs Melanin diskutiert. Die Autoren raten deshalb, Patienten mit Parkinson auch in puncto Haut genau unter die Lupe zu nehmen.

(cd)

Bei Parkinson die Haut im Blick behalten! $\quad \begin{aligned} & \text { Neurology } \\ & 2011,76: 2002\end{aligned}$ (o) Luis Louro / fotolia.com

Kleine Menschen haben geringeres Krebsrisiko

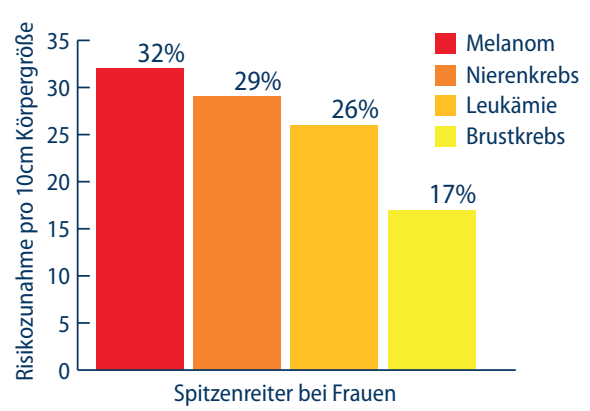

Kleine Personen haben ein signifikant geringeres Risiko, an Krebs zu erkranken als große, so die Ergebnisse einer Studie mit 1.297.124 Frauen. Demnach steigt das Risiko pro $10 \mathrm{~cm}$ mehr an Körpergröße im Durchschnitt um 16\%. Bei Männern erhöht sich das Risiko pro $10 \mathrm{~cm}$ durchschnittlich um $10 \%$.

Lancet Oncology 2011, 12:716

\section{CME-Sommer: Punkte satt und Onkologie}

Es ist eine ernüchternde Nachricht: Das Mammographie-Screening scheint keinen großen Einfluss auf die Brustkrebssterblichkeit zu haben. Das ist das Ergebnis einer aktuellen Studie, bei der die Sterblichkeiten zwischen Ländern verglichen wurde, die das Screening im Abstand von 10-15 Jahren eingeführt hatten. Doch die Studie hat auch etwas Gutes zu bieten: Die Brustkrebssterblichkeit ist in allen untersuchten Ländern deutlich gesunken, zum Teil um bis zu $29 \%$. Bei all der Freude über diese Zahlen sollte man aber den kritischen Blick auf Sinn und Unsinn der Krebsvorsorge nicht vergessen. Lesen Sie in unserem Schwerpunkt, was PSA-Wert, Koloskopie und Co. wirklich bringen. Was Sie außerdem finden: 27 CME-Punkte extra. Denn mit unserer Sommerakademie können Sie ihr Punktekonto füllen - damit der Sommer trotz miesem Wetter doch noch Früchte bringt.

\section{Swanelt hoops}

\section{springermedizin.de}

Top gelesen von

Hausärzten

So reanimiert man heute: Die neuen Leitlinien für die Praxis Herz-Notfall im Flugzeug Sieben Empfehlungen für die COPD-Therapie

Loggen Sie sich ein und nutzen Sie die Fülle von Beiträgen auf springermedizin.de! Die oben genannten Artikel finden Sie, in dem Sie die Schlagworte in die Suche eingeben. 